Pacific Journal of Mathematics

A COMPACT SET THAT IS LOCALLY HOLOMORPHICALLY
CONVEX BUT NOT HOLOMORPHICALLY CONVEX 


\title{
A COMPACT SET THAT IS LOCALLY HOLOMOR- PHICALLY CONVEX BUT NOT HOLOMORPHICALLY CONVEX
}

\section{Michael Freeman and Reese Harvey}

\begin{abstract}
It is shown that a certain simple imbedding $T$ of the ordinary two-dimensional torus in $C^{2}$ contains a polynomially convex compact $T$-neighborhood of each of its points, but $T$ is not holomorphically convex in even the weakest presently accepted sense. This example illustrates some of the limitations of a theory of lower dimensional sets in $C^{n}$. In particular, it shows the difficulty of developing a theory based on local information.
\end{abstract}

In the following $K$ will denote a compact set in $C^{n}, \mathscr{C}(K)$ the Banach algebra of continuous functions on $K$, and $\mathcal{O}(K)$ the algebra of functions holomorphic on some $C^{n}$ neighborhood of $K$. Also, let $A(K)$ denote the Banach subalgebra of $\mathscr{C}(K)$ obtained by taking the closure of the image of $\mathscr{O}(K)$ in $\mathscr{C}(K)$. A compact set $K$ is said to be holomorphically convex if $K$ and the spectrum of $A(K)$ are homeomorphic under the natural map. In [5] a notion of the "envelope of holomorphy", for $K$ a compact subset of $\boldsymbol{C}^{n}$, was introduced; there it was proved, in particular, that $K$ is equal to its envelope if and only if $K$ is holomorphically convex. The Cartan Theorems $\mathrm{A}$ and $B$ for open holomorphically convex sets in $C^{n}$ admit analogues for compact holomorphically convex sets in $C^{n}$ (see [5]). One might conjecture that the E. E. Levi problem for open sets in $C^{n}$ admits a compact analogue. That is, one might conjecture that if $K$ is locally holomorphically convex (i.e., for each point $z \in K$ there exists a compact neighborhood $N$ of $z$ in $K$ such that $N$ is holomorphically convex) then $K$ is holomorphically convex. The example presented below shows that this is not the case.

If "holomorphic approximation" holds on a compact set $K \subset C^{n}$ (i.e., $\mathscr{O}(K)$ is dense in $\mathscr{C}(K)$ ) then the spectrum of $A(K)=\mathscr{C}(K)$ is of course homeomorphic to $K$ so that $K$ is holomorphically convex according to the above definition. Even if a compact set $K$ has the property that "local holomorphic approximation" holds (i.e., for each point $z \in K$ there exists a compact neighborhood $N$ of $z$ in $K$ such that $\mathscr{O}(N)$ is dense in $\mathscr{C}(N)$ ) the set $K$ need not be (globally) holomorphically convex because of the example presented below. In particular, this provides an example of a compact set in $C^{n}$ where local holomorphic approximation holds but global holomorphic approximation does not hold; as distinguished from the well-known 
fact that if $K$ is a compact subset of the complex line $C$ and local holomorphic approximation holds then it is true that global holomorphic approximation holds (see for example [2]).

In [4] a notion of a "totally real set in $C^{n}$ " was introduced in order to better understand the properties of $\boldsymbol{R}^{n}$ in $\boldsymbol{C}^{n}$ which are crucial for the development of Sato's theory of hyperfunctions. Sato's basic theory [12] was shown to hold with $\boldsymbol{R}^{n}$ replaced by a totally real set. In the definition of a compact totally real subset $K$ of $C^{n}$ there are two local requirements which heuristically ensure that $K$ has no (locally) "complex structure of dimension $\geqq 1$ " (see [4], Definition 3.4 and the Remark 1 afterward). The example presented below shows that the local information contained in the assertion that $K$ is a totally real set (which is more than just local holomorphic convexity but less than local holomorphic approximation) is not sufficient to ensure that $K$ is holomorphically convex. In particular, in the duality result, Corollary 3.10 of [4], the hypothesis that $K$ be holomorphically convex is necessary.

We would like to acknowledge that $R$. $O$. Wells has independently verified that the example given here is not holomorphically convex.

The example is very simple. It is just the two-dimensional torus $T$ imbedded in $C^{2}$ as $T=\left\{z:\left(\left|z_{1}\right|-3\right)^{2}+x_{2}^{2}=1, y_{2}=0\right\}$. In fact, (a) the envelope of holomorphy of $T$ is the set

$$
\widetilde{T}=\left\{z:\left(\left|z_{1}\right|-3\right)^{2}+x_{2}^{2} \leqq 1, y_{2}=0\right\}
$$

obtained by filling up $T$ in $\boldsymbol{C} \times \boldsymbol{R} \times\{0\}$; but (b) each point $a$ of $T$ has a compact $T$-neighborhood $N$ on which the polynomials $C\left[z_{1}, z_{2}\right]$ are dense in the Banach space $\mathscr{C}(N)$ of continuous functions on $N$. Of course this implies in particular that each compact subset of $N$ is polynomially and hence holomorphically convex.

The proof of (a) rests on the observation that $T$ has a basis for its neighborhood system consisting of the Hartogs domains

$$
U_{\varepsilon}=\left\{z:\left|\left(\left|z_{1}\right|-3\right)^{2}+x_{2}^{2}-1\right|<\varepsilon,\left|y_{2}\right|<\varepsilon\right\}, \varepsilon>0
$$

(which are clearly circled in $z_{1}$ for each fixed $z_{2}$ ), and on the proposition below, which asserts that the envelope of holomorphy of $U_{\varepsilon}$ is

$$
\widetilde{U}_{\varepsilon}=\left\{z:\left(\left|z_{1}\right|-3\right)^{2}+x_{2}^{2}<1+\varepsilon,\left|y_{2}\right|<\varepsilon\right\}, \frac{1}{2} \geqq \varepsilon>0 .
$$

This shows that any function holomorphic in a neighborhood of $T$ has a holomorphic extension to a neighborhood of $\widetilde{T}$. Moreover, since each $\widetilde{U}_{\varepsilon}$ is holomorphically convex, so is $\widetilde{T}=\bigcap_{\varepsilon>0} \widetilde{U}_{\varepsilon}$. Thus $\widetilde{T}$ is the envelope of holomorphy of $T$ (see [5] for the precise definition of envelope of holomorphy of $T$ ). 
Proposition. $\tilde{U}_{\varepsilon}$ is the envelope of holomorphy of $U_{\varepsilon}$ for $0<$ $\varepsilon \leqq 1 / 2$.

Proof. The open set $\widetilde{U}_{\varepsilon}$ is a domain of holomorphy because it is pseudoconvex (see [3] or [8]). The fact that the functions $z \rightarrow$ $\left(\left|z_{1}\right|-3\right)^{2}+x_{2}^{2}$ and $z \rightarrow\left|y_{2}\right|^{2}$ are plurisubharmonic on $\widetilde{U}_{\varepsilon}, \varepsilon \leqq 1 / 2$, implies that $\widetilde{U}_{\varepsilon}$ is pseudoconvex by [8] Theorem 2.6.7 (iii).

Each function $f$ holomorphic on $U_{\varepsilon}$ has a holomorphic extension to $\widetilde{U}_{\varepsilon}$. For this it suffices to see that the Hartogs-Laurent expansion (see [13] page 130) for $f$ on $U_{\varepsilon}$,

$$
f(z)=\sum_{n=-\infty}^{\infty} f_{n}\left(z_{2}\right) z_{1}^{n},
$$

is normally convergent on $\widetilde{U}_{\varepsilon}$, for then its sum will extend $f$ as asserted. Here the coefficients $f_{n}$ are holomorphic on $\left\{z_{2}: x_{2}^{2}<1+\varepsilon,\left|y_{2}\right|<\varepsilon\right\}$. From the normal convergence of (1) on $U_{\varepsilon}$ it follows that

$$
\sum_{n=-\infty}^{\infty} \sup \left\{\left|f_{n}\left(z_{2}\right) z_{1}^{n}\right|: z \in K_{o}\right\}<\infty,
$$

where $0 \leqq \delta<\varepsilon$ and $K_{\delta}=\left\{z:\left(\left|z_{1}\right|-3\right)^{2}+x_{2}^{2}=1+\delta,\left|y_{2}\right| \leqq \delta\right\}$ (a product of a torus in $\boldsymbol{C} \times \boldsymbol{R}$ and a closed interval in $\boldsymbol{R})$. Now the maximum principle applied (for fixed $z_{2}$ ) to $z_{1} \rightarrow f_{n}\left(z_{2}\right) z_{1}^{n}$ shows that the suprema in (2) become no larger if extended over

$$
\widetilde{K}_{\delta}=\left\{z:\left(\left|z_{1}\right|-3\right)^{2}+x_{2}^{2} \leqq 1+\delta,\left|y_{2}\right| \leqq \delta\right\} .
$$

Thus (2) holds with $K_{\delta}$ replaced by $\widetilde{K}_{\delta}$, and since any compact subset of $\widetilde{U}_{\varepsilon}$ is contained in the interior of some $\widetilde{K}_{\delta}$, the normal convergence of (1) on $\widetilde{U}_{\varepsilon}$ is proved. Thus $\widetilde{U}_{\varepsilon}$ is the envelope of holomorphy of $U_{\varepsilon}$.

Proposition. Each point a of $T$ has a compact neighborhood $N$ in $T$ such that $C\left[z_{1}, z_{2}\right]$ is dense in $\mathscr{C}(N)$.

Proof. Two cases will be distinguished.

(1) The point $a$ is not on one of the top or bottom circles $\left|z_{1}\right|=$ $3, z_{2}= \pm 1+0 i$. Then $a$ is a totally real point of $T$ (i.e., the ordinary real-linear tangent space $T_{a}$ to $T$ at $a$ is not complex-linear). The proposition is known for this case (see [11], [9] or [6]) but a simple direct proof can be based on the real-analyticity of $T$. It will be shown that $a$ has an open neighborhood $U$ such that $U \cap T$ is mapped into $\boldsymbol{R}^{2}$ by a biholomorphic map $\psi=\left(\psi_{1}, \psi_{2}\right): U \rightarrow C^{2}$. Then if $N$ is any compact subset of $T \cap U$, the ordinary Weierstrass Theorem implies that $C\left[w_{1}, w_{2}\right]$ is uniformly dense in $\mathscr{C}(\psi(N))$. Since $\psi$ is invertible, the polynomial combinations of $\psi_{1}, \psi_{2}$ are dense in $\mathscr{C}(N)$. 
If $U$ is taken from the beginning as a polycylinder, then $\psi_{1}$ and $\psi_{2}$ are approximable on $N$ by polynomials in $z_{1}, z_{2}$, which proves the proposition in case (1).

The map \& will be found by constructing its inverse. Note that there is an open neighborhood $V$ of 0 in $R^{2}$ and a real-analytic map $\phi: V \rightarrow T$ such that $\phi(0)=a$ and $d_{0} \phi\left(\boldsymbol{R}^{2}\right)=T_{a}$. Here $d_{f} f$ denotes the Fréchet derivative of $f$ at 0 . Then there is an open set $\tilde{V}$ in $C^{2}$ such that $\tilde{V} \cap \boldsymbol{R}^{2}=V$ and a holomorphic map $\tilde{\phi}: \widetilde{V} \rightarrow \boldsymbol{C}^{2}$ such that $\tilde{\phi} \mid V=\phi$. Clearly, $d_{0} \tilde{\phi}\left(\boldsymbol{R}^{2}\right)=T_{a}$. Moreover, $T_{a} \cap i T_{a}=\{0\}$, so $\boldsymbol{C}^{2}=T_{a}+i T_{a}=$ $d_{0} \tilde{\phi}\left(\boldsymbol{R}^{2}\right)+i d_{0} \tilde{\phi}\left(\boldsymbol{R}^{2}\right)=d_{0} \tilde{\phi}\left(\boldsymbol{R}^{2}+i \boldsymbol{R}^{2}\right)=d_{0} \tilde{\phi}\left(\boldsymbol{C}^{2}\right)$. Thus $d_{0} \tilde{\phi}$ is invertible, so $\tilde{\phi}$ has a holomorphic inverse $\psi$ near 0 by the inverse function theorem.

(2) $\left|a_{1}\right|=3, a_{2}= \pm 1+i 0$. Then there is a closed disk $D=$ $\left\{z_{1}:\left|z_{1}-a_{1}\right| \leqq \varepsilon\right\}$ on which the graph of $g\left(z_{1}\right)=\left(\operatorname{sign} a_{2}\right) \sqrt{1-\left(\left|z_{1}\right|-3\right)^{2}}$ defines a compact set $N=\left\{\left(z_{1}, g\left(z_{1}\right)\right):\left|z_{1}-a_{1}\right| \leqq \varepsilon\right\} \subset T$. Clearly, $N$ is a $T$-neighborhood of $a$. Moreover, the level curves of $g$, as arcs of radii $>1$, do not disconnect $C$ and have no interior points. Therefore, by Mergelyan's Theorem [10] ( $\$ 5$, Theorem 1.5), the polynomial combinations of $z_{1}$ and $g$ are dense in $\mathscr{C}(D)$. The proposition is proved by transporting this property to $N$ via the homeomorphism $z_{1} \rightarrow\left(z_{1}, g\left(z_{1}\right)\right)$.

There is a result (going back to Grauert [3]) of a positive nature which enables one to conclude from local information that a compact subset $K$ of $\boldsymbol{C}^{n}$ is holomorphically convex. Briefly, the method is as follows (cf. [11], [9] or [6]). Suppose that in some $C^{n}$ neighborhood $U_{a}$ of each point $a \in K$ there exists a $C^{2}$ nonnegative strictly plurisubharmonic function $\varphi$ such that $K \cap U_{a}$ equals $\left\{z \in U_{a}: \varphi(z)=0\right\}$. By using a partition of unity one can construct a nonnegative strictly plurisubharmonic function $\phi$ in a neighborhood $U$ of $K$ such that $K=\{z \in U: \varphi(z)=0\}$. Then for sufficiently small $\varepsilon>0$, each of the sets $W_{\varepsilon}=\{z \in U: \varphi(z)<\varepsilon\}$ is a Stein open neighborhood of $K$ and $K$ is $\mathcal{O}\left(W_{\varepsilon}\right)$-convex. Hence $K$ is holomorphically convex. The use of this result is limited by the fact (see [7]) that sets $K$ which satisfy the local condition described above must be (locally) contained in a $\mathscr{C}^{1}$ submanifold of $C^{n}$ all of whose points are totally real.

On the other hand, this technique is extended in [1], where such a function $\varphi$ (which is only required to be plurisubharmonic-not strictly) is constructed in a neighborhood of a point on a two-manifold where its tangent space is complex linear but whose second-order behavior is sufficiently "hyperbolic" (in a precise sense given in [1]). This result is delimited by the above example $T$, which (in the same sense of [1]) exhibits a kind of "parabolic" behavior at such points. 


\section{REFERENCES}

1. M. Freeman, Exceptional points of real submanifolds, (in preparation).

2. T. Gamelin, Uniform Algebras, Prentice Hall, (1969).

3. H. Grauert, On Levi's problem and the imbedding of real-analytic manifolds, Ann. of Math., (2) 68 (1958), 460-472.

4. R. Harvey, The theory of hyperfunctions on totally real subsets of a complex manifold with applications to extension problems, Amer. J. Math., 91 (1969), 853-873.

5. R. Harvey and R. O. Wells, Compact holomorphically convex subsets of a Stein manifold, Trans. Amer. Math. Soc., 136 (1969), 509-516.

6. - Holomorphic approximation on totally real submanifolds of a complex manifold, Math. Ann., (to appear).

7. - Zero sets of nonnegative strictly plurisubharmonic functions, Math. Ann.. (to appear).

8. L. Hormander, An Introduction to Complex Analysis in Several Variables, D. Van Nostrand, Princeton, N. J., (1966).

9. L. Hormander and J. Wermer, Uniform approximation on compact sets in $\boldsymbol{C}^{n}$ Math. Scand., 23 (1968), 5-21.

10. S. N. Mergelyan, Uniform approximation to functions of a complex variable, Amer. Math. Soc. Translation, 101 (1954).

11. R. Nirenberg and R. O. Wells, Approximation theorems on differentiable submanifolds of a complex manifold, Trans. Amer. Math. Soc., 142 (1969), 15-35.

12. M. Sato, Theory of hyperfunctions II, J. Fac. Sci. Univ. Tokyo, Sect. I, 8 (1960), 387-437.

13. V. Vladimirov, Methods of the Theory of Functions of Several Complex Variables, The M. I. T. Press, Cambridge, Mass., (1966).

Received June 22, 1972. The work of author Harvey was supported by NSF grant GP-19011.

UNIVERSITY OF KENTUCKY

AND

Rice University and the Institute for Advanced Study 



\section{PACIFIC JOURNAL OF MATHEMATICS}

\section{EDITORS}

RICHARD ARENS (Managing Editor)

University of California

Los Angeles, California 90024

R. A. Beaumont

University of Washington

Seattle, Washington 98105
J. DUGUNDJI*

Department of Mathematics

University of Southern California

Los Angeles, California 90007

D. Gilbarg and J. Milgram

Stanford University

Stanford, California 94305

\section{ASSOCIATE EDITORS}
E. F. BECKENBACH
B. H. NeumanN
F. WOLF
K. YosHIDA

\section{SUPPORTING INSTITUTIONS}

UNIVERSITY OF BRITISH COLUMBIA
CALIFORNIA INSTITUTE OF TECHNOLOGY
UNIVERSITY OF CALIFORNIA
MONTANA STATE UNIVERSITY
UNIVERSITY OF NEVADA
NEW MEXICO STATE UNIVERSITY
OREGON STATE UNIVERSITY
UNIVERSITY OF OREGON
OSAKA UNIVERSITY

UNIVERSITY OF BRITISH COLUMBIA

UNIVERSITY OF CALIFORNIA

MONTANA STATE UNIVERSITY

UNIVERSITY OF NEVADA

OREGON STATE UNIVERSITY

OSAKA UNIVERSITY
UNIVERSITY OF SOUTHERN CALIFORNIA

STANFORD UNIVERSITY

UNIVERSITY OF TOKYO

UNIVERSITY OF UTAH

WASHINGTON STATE UNIVERSITY

UNIVERSITY OF WASHINGTON

\section{AMERICAN MATHEMATICAL SOCIETY} NAVAL WEAPONS CENTER

* C. R. DePrima California Institute of Technology, Pasadena, CA 91109, will replace J. Dugundji until August 1974. 


\section{Pacific Journal of Mathematics}

\section{Vol. 48, No. $1 \quad$ March, 1973}

Jan Aarts and David John Lutzer, Pseudo-completeness and the product of Baire

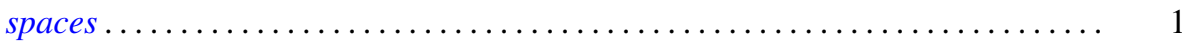

Gordon Owen Berg, Metric characterizations of Euclidean spaces ............ 11

Ajit Kaur Chilana, The space of bounded sequences with the mixed topology ..... . 29

Philip Throop Church and James Timourian, Differentiable open maps of

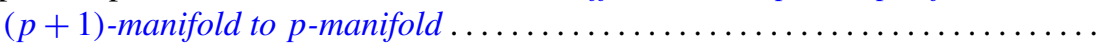

P. D. T. A. Elliott, On additive functions whose limiting distributions possess a finite

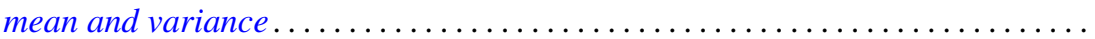

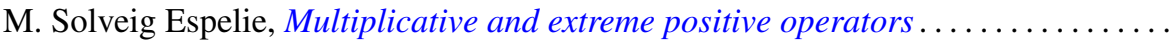

Jacques A. Ferland, Domains of negativity and application to generalized convexity

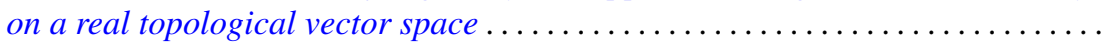

Michael Benton Freeman and Reese Harvey, A compact set that is locally holomorphically convex but not holomorphically convex ...............

Roe William Goodman, Positive-definite distributions and intertwining

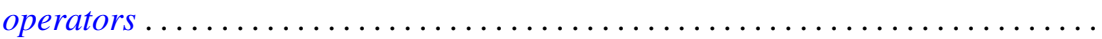

Elliot Charles Gootman, The type of some $C^{*}$ and $W^{*}$-algebras associated with

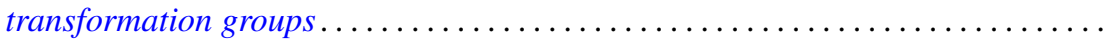

David Charles Haddad, Angular limits of locally finitely valent holomorphic

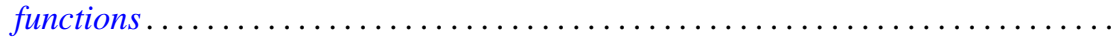

William Buhmann Johnson, On quasi-complements .

William M. Kantor, On 2-transitive collineation groups of finite projective spaces...

Joachim Lambek and Gerhard O. Michler, Completions and classical localizations of right Noetherian rings

Kenneth Lamar Lange, Borel sets of probability measures ......

David Lowell Lovelady, Product integrals for an ordinary differential equation in a Banach space

Jorge Martinez, A hom-functor for lattice-ordered groups .........

W. K. Mason, Weakly almost periodic homeomorphisms of the two sphere ....

Anthony G. Mucci, Limits for martingale-like sequences .......

Eugene Michael Norris, Relationally induced semigroups ...

Arthur E. Olson, A comparison of c-density and $k$-density ......

Donald Steven Passman, On the semisimplicity of group rings of linear groups.

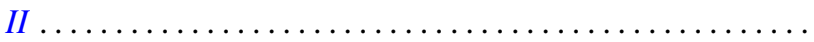

Charles Radin, Ergodicity in von Neumann algebras .

P. Rosenthal, On the singularities of the function generated by the Bergman operator of the second kind.

Arthur Argyle Sagle and J. R. Schumi, Multiplications on homogeneous spaces,

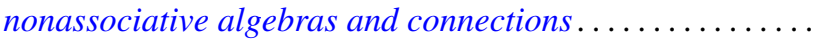

Leo Sario and Cecilia Wang, Existence of Dirichlet finite biharmonic functions on

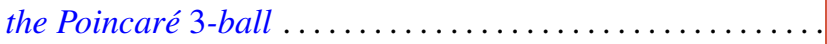

Ramachandran Subramanian, On a generalization of martingales due to Blake ..

Bui An Ton, On strongly nonlinear elliptic variational inequalities.

Seth Warner, A topological characterization of complete, discretely valued

fields. 